\title{
O uso da artéria torácica interna no idoso: indicações e resultados imediatos
}

\author{
André Luiz TYSZKA* e Leila Satomi FUCUDA*
}

RBCCV 44205-555

Tyszka A L \& Fucuda L S - O uso da artéria torácica interna no idoso: indicações e resultados imediatos. Rev Bras Cir Cardiovasc 2001; 16(4): 269-74.

RESUMO: Objetivo: A artéria torácica interna é o enxerto de eleição para revascularização do ramo interventricular anterior. Entretanto, a incidência de sua utilização nos pacientes idosos acima de 70 anos, varia amplamente nos Serviços. Este estudo revisa as indicações de sua utilização nesta população e apresenta os resultados imediatos obtidos em nosso Serviço.

Casuística e Métodos: Foram analisados 163 pacientes, dos quais 40 (24,53\%) possuíam mais de 70 anos (Grupo 2), revascularizados no período de julho de 1999 a dezembro de 2000. Os dados pré-operatórios avaliados foram sexo, fatores de risco para aterosclerose, classe funcional, classificação da angina, infarto prévio e função ventricular esquerda. Os dados operatórios estudados foram número de vasos revascularizados, tipos de enxertos, e intercorrências cirúrgicas. Foram também analisadas as complicações pós-operatórias até 30 dias.

Resultados: Todos os ramos interventriculares anteriores dos pacientes do Grupo 1 foram revascularizados com a artéria torácica interna e 95\% dos pacientes do Grupo 2 receberam este enxerto. Não houve diferença estatística entre as características pré e pós-operatórias entre os dois grupos.

Conclusão: Embora os pacientes idosos apresentem maior risco de complicações cirúrgicas, a utilização da artéria torácica interna não traz aumento da morbidade e inclusive melhora a sobrevida precoce e tardia, devendo portanto ser considerada como enxerto de primeira escolha para esta população.

DESCRITORES: Coronariopatia, cirurgia. Revascularização miocárdica, idoso. Artéria torácica interna, cirurgia. Procedimentos cirúrgicos cardíacos, métodos, idoso.

\section{INTRODUÇÃO}

Dados demográficos recentes demonstram que a população de idosos no Brasil, seguindo a tendência dos países desenvolvidos, vem apresentando constante crescimento (www.datasus.gov.br). Com melhores acessos aos sistemas de saúde e a grande prevalência de doenças ateroscleróticas dos países ocidentais, atenção especial deve ser dada a esta parcela da população que necessita de tratamento para insuficiência coronária. Hoje, esses pacientes representam cerca de $20 \%$ dos doentes encaminhados para a revascularização miocárdica cirúrgica $(1,2)$.

As indicações de revascularização miocárdica no idoso, bem como a segurança do procedimento cirúrgico, estão bem estabelecidas. Segundo IGLÉZIAS et al. (3) revendo 361 pacientes com mais de 70 anos, revascularizados entre 1992 e 1995, este procedimento pode ser realizado com baixa morbimortalidade, proporcionando substancial

Trabalho realizado no Hospital Santa Rita de Maringá. Maringá, PR, Brasil.

Recebido para publicação em maio de 2001.

* Do Instituto do Coração de Maringá.

Endereço para correspondência: André Luiz Tyszka. Av. Independência, 32. Maringá, PR, Brasil. CEP: 87015-020 Tel./Fax: (44) 225-7333.

e-mail:altyska@uol.com.br 
Tyszka A L \& Fucuda L S - O uso da artéria torácica interna no idoso: indicações e resultados imediatos. Rev Bras Cir Cardiovasc 2001; 16(4):269-74.

melhora da qualidade de vida e devendo ter as mesmas indicações cirúrgicas dos pacientes mais jovens. BUSH et al. (2) demonstraram que os pacientes idosos, embora apresentem maiores chances de complicações, podem ser operados com riscos bastante aceitáveis e que a revascularização miocárdica aumenta a expectativa e a qualidade de vida, além de reduzir os riscos de morte por causa cardíaca. Outros trabalhos da literatura mundial também corroboram estas afirmações (4-6).

Conforme apontado por GARDNER et al. (1) e BUSH et al. (2), mesmo com a maior utilização dos enxertos de artéria torácica interna (ATI) nos últimos anos, o seu emprego ainda é bastante variável. $\mathrm{Na}$ literatura nacional, SILVA et al. ${ }^{(7)}$, em trabalho publicado em 1997, utilizaram a ATI em 43,9\% dos pacientes e ALMEIDA et al. (8), em divulgação de 2000, em $46 \%$ dos idosos revascularizados. Já DENINGER et al. (9), em 1999, utilizaram em 87,5\%, embora relatem que mais recentemente esta incidência tenha aumentado para $98,9 \%$ dos casos. A incidência demonstrada por KUBRUSLY et al. (10), em 2000, foi de $92 \%$ de seus pacientes. Outros autores, como IGLÉZIAS et al. (3) e LOURES et al. (11), que descreveram as suas experiências em 1999, com a revascularização em idosos, não relataram a proporção do uso de enxertos arteriais.

Reportamos nossa experiência recente com o uso da ATIE em idosos para documentar os resultados operatórios e estabelecer a segurança e a importância de sua utilização como fator prognóstico. A análise destes dados visaram avaliar os resultados imediatos quanto à morbimortalidade dos pacientes septuagenários nos quais foi utilizada a artéria torácica interna esquerda e relacionar com pacientes de faixa etária inferior, revisando também a literatura específica.

\section{CASUÍSTICA E MÉTODOS}

Foram analisados consecutivamente os pacientes submetidos à revascularização miocárdica isolada (primária ou reoperação) no período de julho de 1999 a dezembro de 2000 pelo Instituto do Coração de Maringá do Hospital Santa Rita.

Dos 163 pacientes operados, 123 (75,47\%) possuíam menos de 70 anos (Grupo 1) e 40 $(24,53 \%)$ possuíam 70 anos ou mais, formando o Grupo 2. Os dados demográficos dos pacientes encontram-se resumidos na Tabela 1. A idade global variou de 36 a 83 anos com média de $61,73$ anos (DP $=9,58)$. No Grupo 1 , dos pacientes com menos de 70 anos, a idade média foi de 58,02 anos e no grupo 2 foi de 73,15 anos.
TABELA 1

\begin{tabular}{lcc}
\hline \multicolumn{3}{c}{ DADOS DEMOGRÁFICOS } \\
\hline VARIÁVEL & GRUPO 1 (N) & GRUPO 2 (N) \\
\hline Idade média (anos) & 58,02 & 73,15 \\
Sexo feminino (\%) & $39,83 \%$ & $35 \%$ \\
Uso da ATI & $120(97,56 \%)$ & $38(95 \%)$ \\
\hline
\end{tabular}

ATI: artéria torácica interna

Dos pacientes do Grupo 1, com menos de 70 anos, 120 (97,56\%) receberam pelo menos uma artéria torácica interna, sendo que todas as vezes que o ramo interventricular anterior necessitou ser revascularizado recebeu enxerto de artéria torácica interna esquerda. Nos 40 pacientes do Grupo 2, $95 \%$ foram revascularizados com a artéria torácica interna. Cinco pacientes do Grupo 1 receberam mais de um enxerto arterial, 3 foram revascularizados com duas artérias torácicas internas e 2 com a artéria radial.

\section{Técnica Operatória}

Com o paciente devidamente anestesiado, preparado e monitorizado, procedia-se a esternotomia mediana e a dissecção e preparo da artéria torácica interna esquerda. Simultaneamente, a veia safena magna foi dissecada por técnica anteriormente descrita (12). A circulação extracorpórea foi instalada após a heparinização (3 $\mathrm{mg} / \mathrm{kg}$ de peso), com canulação da aorta ascendente e átrio direito e o paciente mantido em hipotermia sistêmica moderada (32 graus). A proteção miocárdica foi realizada com cardioplegia sangüínea gelada (4 graus), via anterógrada, multidose, a cada 15 minutos e resfriamento miocárdico tópico com solução salina gelada intermitente. As anastomoses distais foram realizadas com fio de polipropilene 7-0 e as anastomoses proximais, através de pinçamento aórtico parcial com fio de polipropilene 6-0, durante a fase de reaquecimento. Após a retirada da circulação extracorpórea e neutralização da heparina com sulfato de protamina (1:1), as cavidades pleural esquerda e mediastinal foram drenadas e o tórax fechado por planos de maneira habitual.

Em 6 pacientes do Grupo 1 e em 3 do Grupo 2, submetidos a revascularização uniarterial, ou seja, artéria torácica interna esquerda para o ramo interventricular anterior, o procedimento foi realizado sem a utilização da circulação extracorpórea. 
Tyszka A L \& Fucuda L S - O uso da artéria torácica interna no idoso: indicações e resultados imediatos. Rev Bras Cir Cardiovasc 2001; 16(4):269-74.

Os dados pré, intra e pós-operatórios foram coletados em todos os pacientes. Detalhes da história médica, incluindo infarto miocárdico prévio, natureza da angina de peito, fatores de risco para doença coronariana e doenças associadas, foram anotados. A extensão do envolvimento coronário e a função ventricular esquerda pré-operatória determinadas pela cineangioventriculografia também foram anotadas. Informações intra-operatórias analisadas incluíram número e qualidade dos enxertos, e intercorrências cirúrgicas. Informações pós-operatórias avaliadas foram: incidência de reoperação por sangramento, incidência de infecção esternal (definida como infecção da ferida operatória abaixo ou envolvendo o esterno), necessidade de suporte inotrópico por drogas ou balão intra-aórtico, insuficiência respiratória (definida como necessidade de ventilação por mais de 24 horas ou reintubação), insuficiência renal aguda (definida como aumento do dobro da creatinina pré-operatória ou necessidade de diálise), incidência de arritmias, tempo de permanência na UTI e hospitalar e mortalidade hospitalar (definida como morte ocorrida dentro de 30 dias de pós-operatório, ou a qualquer tempo durante hospitalização contínua desde a operação). Os pacientes foram acompanhados ambulatorialmente durante período de 30 dias de pós-operatório.

Para a análise estatística dos dados nominais utilizou-se o teste exato de Fisher (STATISTIC 5.0 StatSoft, Inc. Tulsa, OK, EUA 1995. Windows). O nível de significância estabelecido para a análise foi de 0,05 ou $5 \%$.

\section{RESULTADOS}

\section{Dados Pré-Operatórios}

Os dados pré-operatórios dos dois grupos estão relacionados na Tabela 2. Não houve diferença significativa entre as variáveis analisadas.

\section{Dados Operatórios e Pós-Operatórios}

Os dados operatórios e pós-operatórios dos dois grupos estão relacionados na Tabela 3. Não se encontrou diferença significativa entre os dois grupos.

A mortalidade global foi de 3,68\%, (Grupo $1=$ $2,43 \%$ e Grupo $2=7,5 \%$ ). Analisando os pacientes com mais de 70 anos e se excluindo os pacientes que não receberam enxerto de artéria torácica interna, a mortalidade foi de $5 \%$, pois dos 3 óbitos 1 paciente não havia recebido enxerto arterial.
TABELA 2

\begin{tabular}{lcc}
\hline \multicolumn{3}{c}{ DADOS PRÉ-OPERATÓRIOS } \\
\hline VARIÁVEL & GRUPO 1 & GRUPO 2 \\
\hline IAM prévio & $32(26,02 \%)$ & $11(27,5 \%)$ \\
\hline Cirurgia coronária prévia & $2(1,62 \%)$ & 0 \\
Fração de ejeção(<30\%) & $19(15,44 \%)$ & $6(15 \%)$ \\
Hipertensão arterial & $38(30,8 \%)$ & $5(12,5 \%)$ \\
Angina instável & $17(13,8 \%)$ & $9(22,5 \%)$ \\
\hline Tabagismo & $52(42,2 \%)$ & $13(32,5 \%)$ \\
Obesidade & $14(11,38 \%)$ & $3(7,5 \%)$ \\
Diabete melito & $36(29,26 \%)$ & $10(25 \%)$ \\
Doença vascular cerebral & $3(2,43 \%)$ & $1(2,5 \%)$ \\
Doença vascular periférica & $12(9,75 \%)$ & $2(5 \%)$ \\
Outras doenças & $6(4,87 \%)$ & $2(5 \%)$ \\
\hline
\end{tabular}

TABELA 3

\begin{tabular}{lcc}
\hline \multicolumn{3}{c}{ DADOS OPERATÓRIOS E PÓS-OPERATÓRIOS } \\
\hline DADOS VARIÁVEIS & GRUPO 1 & GRUPO 2 \\
\hline Número de enxertos (m) & 3,00 & 2,92 \\
Uso de Balão intra-aórtico (\%) & $4(3,25 \%)$ & $1(2,5 \%)$ \\
Arritmias (\%) & $4(3,25 \%)$ & $2(5 \%)$ \\
Reoperação por sangramento (\%) & $1(0,81 \%)$ & $1(2,5 \%)$ \\
Infecção esternal (\%) & $2(1,62 \%)$ & $1(2,5 \%)$ \\
AVC (\%) & $3(2,43 \%)$ & $2(5 \%)$ \\
Insuficiência renal (\%) & $3(2,43 \%)$ & $1(2,5 \%)$ \\
Insuficiência respiratória (\%) & $1(0,81 \%)$ & $2(5 \%)$ \\
IAM pós-operatório (\%) & $1(0,81 \%)$ & 0 \\
Mortalidade (\%) & $3(2,4 \%)$ & $3(7,5 \%)$ \\
Tempo de internação em UTI (m) & 2,6 dias & 3,2 dias \\
Tempo de internação hospitalar (m) & 6,4 dias & 8,6 dias \\
\hline
\end{tabular}

$\mathrm{m}=$ média; $\mathrm{IAM}=$ infarto agudo do miocárdio; $\mathrm{AVC}=$ acidente vascular cerebral; UTI = unidade de terapia intensiva.

\section{COMENTÁRIOS}

A artéria torácica interna (ATI) é o melhor enxerto para revascularização miocárdica em termos de patência a longo prazo, livre recorrência de angina e

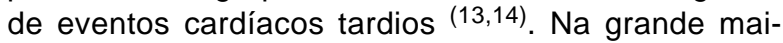
oria dos Serviços tem sido utilizada como enxerto de primeira escolha desde $1986{ }^{(14)}$. Devido ao aparente benefício da utilização da ATI, tenha sido mais enfatizado quanto aos resultados tardios de patência e sobrevida, muitos cirurgiões têm evitado seu uso nos pacientes idosos, assumindo não estar acrescentando substancial benefício a esta população ${ }^{(13)}$. 
Tyszka A L \& Fucuda L S - O uso da artéria torácica interna no idoso: indicações e resultados imediatos. Rev Bras Cir Cardiovasc 2001; 16(4):269-74.

Nos EUA, a expectativa de vida atual é de aproximadamente 72 anos, porém as pessoas que alcançam a idade de 65 anos têm em média mais 15,6 anos de vida; quando atingem a idade de 75 anos têm expectativa de mais 9,8 anos e os que chegam aos 80 anos têm expectativa estimada em mais 7 anos de vida (4). Portanto, mais importante que a expectativa de vida global da população geral é a expectativa de vida para quem atinge os 70 anos.

Considerando, desta maneira, a expectativa de vida dos pacientes idosos, a utilização da ATI deve ser estimulada. A sobrevida de 5 anos, dos pacientes acima de 70 anos submetidos a revascularização cirúrgica do miocárdio, é comparável com a curva de sobrevida atuarial dos pacientes da quinta e sexta década de vida, e é maior quando comparada com a população geral de mesma idade, sexo e etnia, conforme demonstrado por HORNEFFER et al. (15) e por CANE et al. (16). FRUITMAN et al. (17), em 1999, e KHAN et al. (18), em 2000, estudaram o impacto da revascularização miocárdica sobre a qualidade de vida e concluíram que a diminuição da freqüência de episódios anginosos e a melhora da capacidade funcional coloca estes pacientes em situação igual ou melhor que a população geral de mesma idade.

Os enxertos venosos apresentam grande diminuição da patência ao redor do sétimo ano após a revascularização, com retorno, principalmente, à angina de peito (19). A principal causa de necessidade de reintervenção é a aterosclerose dos enxertos venosos, mais do que a progressão da doença no leito arterial nativo (20). Portanto, a não utilização da ATI no idoso, devido à presumida perda de importância da sua patência tardia, não pode ser vista como correta.

A patência imediata é melhor com a utilização da ATI esquerda revascularizando o ramo interventricular anterior, quando comparada à veia safena ${ }^{(21)}$ e isto é de maior importância quando consideramos a grande incidência de enxertos venosos de baixa qualidade, principalmente em pacientes do sexo feminino idosas (1).
Com os resultados publicados com o uso da ATI, houve uma ampliação do seu uso, principalmente nos pacientes mais idosos, do sexo feminino e nas operações de emergência. Idade, sexo, envolvimento de tronco da artéria coronária esquerda e função ventricular esquerda deprimida não deveriam ser mais considerados relevantes na indicação ou não do uso da ATI. Contra-indicações relativas são em pacientes com irradiação da parede anterior do tórax, lesões do tronco braquiocefálico ou da artéria subclávia e nas reoperações onde a substituição de um enxerto venoso patente poderia resultar em síndrome de má perfusão (22).

Embora o paciente idoso possua algumas particularidades que desestimulam a utilização dos enxertos arteriais, como o maior número de enxertos necessários e a maior morbidade geral, não se encontrou, na literatura, fatores que demonstrassem aumento da morbi-mortalidade em pacientes nos quais foi utilizada a ATI. GARDNER et al. (2), comparando pacientes idosos revas-cularizados sem ATI com os pacientes que receberam esse enxerto arterial, pode demonstrar significativa redução da mortalidade hospitalar e apontou a utilização da ATI como fator preditivo independente para a melhor sobrevida precoce e tardia.

Este estudo não teve como objetivo a comparação direta entre os pacientes jovens e idosos. Já está bem estabelecido por estudos com casuísticas maiores que os pacientes idosos estão mais sujeitos às complicações pós-operatórias, embora possuam as mesmas indicações para revascularização miocárdica que os doentes jovens $(3,4)$. Visou-se, através dos nossos resultados e da literatura revisada, demonstrar que a utilização da ATI de rotina não acrescenta aumento na incidência das complicações cirúrgicas e com isto estabelecer a importância de sua utilização.

O presente estudo propõe como segura a utilização de rotina da artéria torácica interna para os pacientes idosos, devendo ser considerada como enxerto de primeira escoIha, mesmo para essa população. 
Tyszka A L \& Fucuda L S - O uso da artéria torácica interna no idoso: indicações e resultados imediatos. Rev Bras Cir Cardiovasc 2001; 16(4):269-74.

RBCCV 44205-555

Tyszka A L \& Fucuda L S - The use of the internal mammary artery in the elderly: indications and immediate outcome. Rev Bras Cir Cardiovasc 2001; 16(4): 269-74

ABSTRACT: Objective: The internal mammary artery (IMA) is the best conduit for use as a descending anterior artery bypass graft. The incidence of the IMA use in the elderly is widely variable. This study reviews the indications of the coronary artery bypass grafting with the internal mammary artery in the elderly and shows our experience with this procedure.

Methods: From July 1999 through December 2000, 163 patients underwent isolated coronary artery bypass grafting. Of these, 40 patients (24.53\%) were 70 years or older (Group 2). The preoperative data included gender, coronary risk factors, NYHA functional class, angina class, history of previous myocardial infarction, and left ventricular function. Intraoperative information included the number of graft performed, whether the IMA was used, and incidence of surgical complications. The incidence of the postoperative complications was recorded in all patients.

Results: In the Group 1 all anterior interventricular branch was revascularized with IMA, and in the Group 2, 95\% received the IMA graft. No significant differences were noted in the preoperative and postoperative data between the two groups.

Conclusion: Although elderly patients requiring bypass grafting clearly are at increased risk of the surgical complications, an IMA graft does not increase the morbidity and appears to improve both early and late survival, making it a first selection graft for such a population.

DESCRIPTORS: Coronary disease, surgery. Myocardial revascularization, aged. Internal mammary artery, surgery. Cardiac surgical procedures, methods, aged.

\section{REFERÊNCIAS BIBLIOGRÁFICAS}

1 Gardner T J, Greene P S, Rykiel M F et al. - Routine use of the left mammary artery graft in the elderly. Ann Thorac Surg 1990; 49:188-93.

2 Busch T, Friedrich M, Sirbu H, Stamm C, Zenker D, Dalichau $\mathrm{H}$ - Coronary artery bypass procedures in septuagenarians are justified: short and longterm results. J Cardiovasc Surg (Torino)1999; 40: 83-91.

3 Iglézias J C R, Oliveira Jr J L, Fels K W et al. - Fatores prognósticos na revascularização do miocárdio em pacientes idosos. Rev Bras Cir Cardiovasc 1997; 12: 325-34.

4 Loop F D, Lytle B W, Cosgrove D M et al. - Coronary artery bypass graft surgery in the elderly. Indications and outcome. Cleve Clin J Med 1988; 55: $23-34$

5 Santibanez E F, Rendon, E F, Ramirez M S, Lopez S F, Barragan G R - Left internal mammmary artery in octagenarians: is its use in coronary artery revascularization justified? Arch Inst Cardiol Mex 1997; 67: 414-8.

6 Talwalkar N G, Damus P S, Durban L H et al. - Outcome of isolated coronary artery bypass surgery in octogenarians. J Card Surg 1996; 11: 172-9.
7 Silva L H F, Nascimento C S, Viotti Jr L A P Revascularização miocárdica em idosos. Rev Bras Cir Cardiovasc 1997; 12: 2-16.

8 Almeida R M S, Lima Jr J D, Martins J F, Loures D R R - Revascularização do miocárdio em pacientes após a oitava década de vida. In: Anais do $28^{\circ}$ Congresso Paranaense de Cardiologia. Ponta Grossa, PR: Sociedade Paranaense de Cardiologia, 2000: 35.

9 Deninger $\mathrm{M} O$, Oliveira $O \mathrm{G}$, Guedes $\mathrm{M}$ G A et al. Cirurgia de revascularização miocárdica no idoso: estudo descritivo de 144 casos. Rev Bras Cir Cardiovasc 1999; 14: 226-32.

10 Kubrusly L F, Martins A F, Madeira J N et al. - Uso da artéria mamária interna esquerda para revascularização de pacientes idosos. In: Anais do $28^{\circ}$ Congresso Paranaense de Cardiologia. Ponta Grossa, PR: Sociedade Paranaense de Cardiologia, 2000: 94.

11 Loures D R R, Carvalho R G, Mulinari L A et al. Cirurgia cardíaca no idoso. Rev Bras Cir Cardiovasc 2000; 15: 1-5.

12 Tyszka A L - Obtenção da veia safena magna através de dissecção minimamente invasiva para enxerto venoso nas revascularizações miocárdicas: resultados preliminares. [Dissertação. Mestrado] Curitiba: Universidade Federal do Paraná, 1999. 61p. 
Tyszka A L \& Fucuda L S - O uso da artéria torácica interna no idoso: indicações e resultados imediatos. Rev Bras Cir Cardiovasc 2001; 16(4):269-74.

Barner H B, Swartz M T, Mudd J G, Tyras D H - Late patency of the internal mammary artery as a coronary bypass conduit. Ann Thorac Surg 1982; 34: 408-12.

14 Loop F D, Lytle B W, Cosgrove D M et al. - Influence of the internal-mammary-artery graft on 10-year survival and the other cardiac events. $N$ Engl $J$ Med 1986; 314: 1-6.

Horneffer P J, Gardner T J, Manolio T A et al. - The effects of age on outcome after coronary bypass surgery. Circulation 1987; 76 (5 pt 2): V6-12.

16 Cane M E, Chen C, Bailey B M et al. - CABG in octagenarians: early and late events and actuarial survival in comparison with a matched population. Ann Thorac Surg 1995; 60:1033-7.

17 Fruitman D S, MacDougall C E, Ross B - Cardiac Surgery in octagenarians: can elderly patients benefit? Quality of life after cardiac surgery. Ann Thorac Surg 1999; 68: 129-35.
18 Khan J H, Magnetti S, Davis E, Zhand J - Late outcomes of open heart surgery in patient 70 years or older. Ann Thorac Surg 2000; 69:165-70.

19 Loop F D, Lytle B W, Cosgrove D M et al. - Reoperation for coronary atherosclerosis: changing practice in 2509 consecutive patients. Ann Surg 1990; 212: 378-5.

20 Cosgrove D M, Loop F D, Lytle B W et al. - Predictors of reoperation after myocardial revascularization. $J$ Thorac Cardiovasc Surg 1986; 92: 811-21.

21 Fitzgibbon G M, Kafka H P, Leach A J, Keon W J, Hooper G D, Burton J R - Coronary bypass graft fate and patient outcome: angiographic follow-up of 5,065 grafts related to survival and reoperation in 1,388 patients during 25 years. J Am Coll Cardiol 1996; 28: 616-26.

22 Loop F D - Coronary artery surgery: the end of the beginning. Eur J Cardiothorac Surg 1998; 14: 554-71. 